\title{
Gastrointestinal involvement in systemic sclerosis: Effects on morbidity and mortality and new therapeutic approaches
}

\author{
Anna-Maria Hoffmann-Vold ${ }_{1,2}$ and Elizabeth R. Volkmann 3
}

\begin{abstract}
The majority of research studies in systemic sclerosis focus largely on addressing skin and cardiopulmonary manifestations. Fewer studies assess the pathogenesis and treatment of gastrointestinal tract involvement in systemic sclerosis, despite the fact that the majority of patients with systemic sclerosis have gastrointestinal manifestations and these manifestations are a leading cause of death in systemic sclerosis. The present review provides a comprehensive update on morbidity and mortality outcomes related to gastrointestinal involvement in systemic sclerosis. This review also describes conventional and emerging approaches to managing gastrointestinal symptoms in systemic sclerosis. Recent developments in systemic sclerosis-gastrointestinal research efforts have revealed promising treatment targets, including specific auto-antibodies and microbiota alterations. This review will conclude with an overview of future research directions that may improve our understanding of systemic sclerosis-gastrointestinal involvement and ultimately help to alleviate suffering from this devastating dimension of systemic sclerosis.
\end{abstract}

\section{Keywords}

Systemic sclerosis, scleroderma, gastrointestinal, motility, microbiota

\section{Gastrointestinal involvement in systemic sclerosis}

Systemic sclerosis ( $\mathrm{SSc}$ ) is a connective tissue disease with multisystem involvement; the gastrointestinal (GI) tract is affected in up to $90 \%$ of SSc patients.1,2 The fundamental mechanisms underlying the pathogenesis of SSc-GI manifestations likely involve inflammation, autoimmunity, fibrosis, and vasculopathy; however, additional factors such as medications, GI microbiota, diet, and other comor-bidities are likely contributory.3 Any part of the GI tract, from the mouth to the anal sphincter, can be afflicted in SSc, and symptoms, disease severity, and clinical impact vary based on the location of involvement and degree of impairment.4 Overall, the most commonly reported GI involvements are as follows: (1) reduced motility of the esophagus with relaxation of the lower esophageal sphinc-ter, (2) gastroesophageal reflux disease (GERD), (3) lower intestinal dysmotility leading to bloating, diarrhea, and/or constipation, (4) small intestine malabsorption, (5) small intestinal bacterial overgrowth (SIBO), and (6) fecal incontinence.5,6 Some SSc patients only have upper or lower GI involvement, while others show involvement of both.2 GI involvement is severe if pseudo-obstruction, malabsorp-tion, malnutrition, and/or $10 \%$ weight loss in association with the use of antibiotics for bacterial overgrowth and/or esophageal stricture are present.7 A recent report from patients with very early SSc showed that GI involvement in the form of esophageal and anal involvement was already

\footnotetext{
1Department of Rheumatology, Oslo University

Hospital, Rikshospitalet, Oslo, Norway

2Institute of Clinical Medicine, University of Oslo, Oslo, Norway

${ }_{3}$ Division of Rheumatology, Department of Medicine, University

of California-Los Angeles (UCLA), Los Angeles, CA, USA
}

Corresponding author:

Anna-Maria Hoffmann-Vold, Department of Rheumatology,

Oslo University Hospital, Rikshospitalet, $\mathrm{Pb} 4950$ Nydalen,

0424 Oslo, Norway.

Email: a.m.hoffmann-vold@medisin.uio.no 


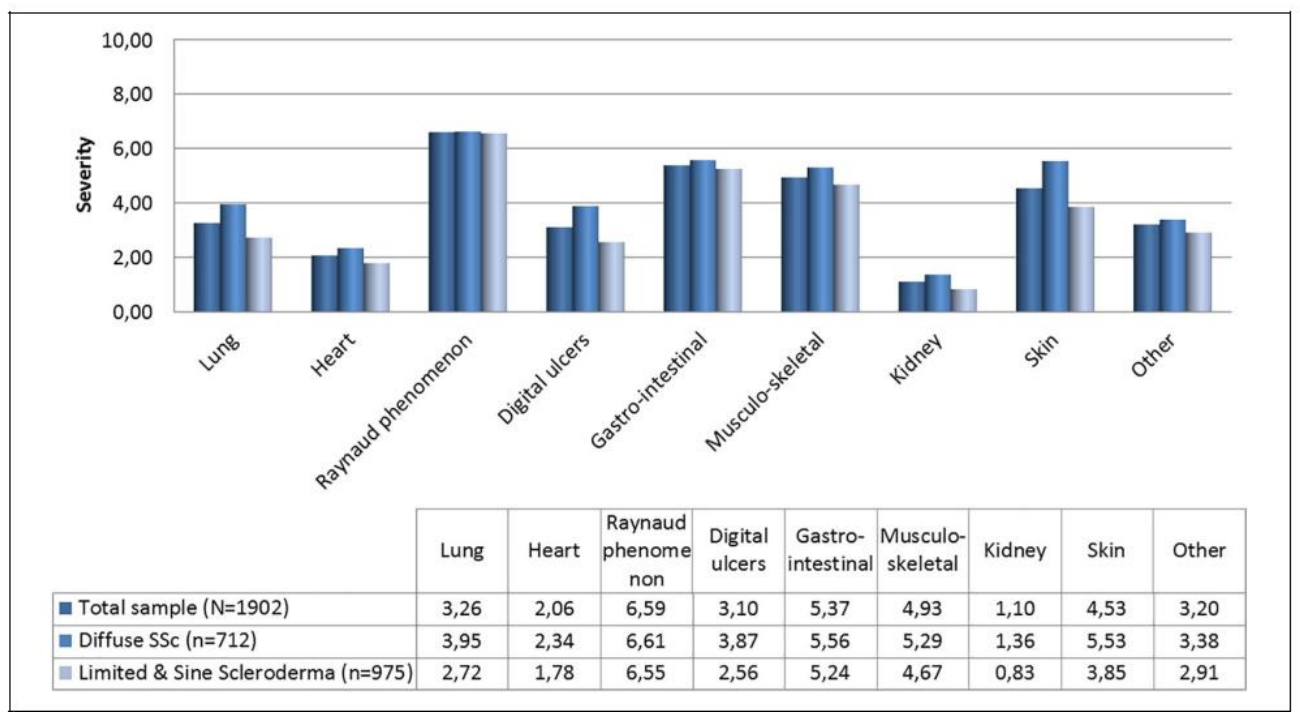

Figure 1. The impact of organ manifestations on systemic sclerosis patients' daily life.

The gastrointestinal tract was one of the organ systems which had the greatest impact on severity assessment reported by SSc patients. The impact of organ involvement was assessed on a scale ranging from 0 (no impact) to 10 (extremely severe impact). 12

present within 6 years after the onset of Raynaud's phenomenon.8 Also, the probability of severe GI disease early in the course of disease is high, with an estimated preva-lence of $9 \%$ at 2 years and $16 \%$ at 4 years. 9

GI symptoms often progress over the disease course, but to date, there are no valid biomarkers for disease progression or risk stratification.10 In a recent report, severe GI disease was associated with inflammatory myositis, telangiectasia, and modified Rodnan skin score.9 In another report of $66 \mathrm{SSc}$ patients with severe GI involvement, the clinical features strongly associated with severe GI dysmotility included male sex, myopathy, and sicca symptoms.10 In a multivariate analysis, there was no significant association between severe GI dysmotility and the presence of SSc-specific auto-antibodies, including anti-topoisomerase 1, anti-RNA polymerase III (although both were present significantly less often in patients with severe GI impairment in univariate analyses), or anti-centromere antibodies.10 In another study, anti-RNPC3 antibody--positive SSc patients were more likely to have moderate-to-severe GI dysfunction and esophageal dysmotility was more prevalent among anti-RNPC3-positive patients with SSc.11 As previous studies on both associations and predictive value of antibodies for GI disease in SSc have shown conflicting results, further longitudinal studies are needed to evaluate the role of anti-bodies and clinical variables in GI involvement in SSc patients.

\section{Effects of Gl involvement on morbidity in SSc}

GI involvement severely affects quality of life in SSc. In a recent large international SSc patient survey including
1902 patients from 60 countries, GI involvement had the greatest impact on health-related quality of life (HRQoL) in patients with SSc on a daily basis and was together with Raynaud's phenomenon the factor associated with the most negative perception of illness severity, as shown in Figure 1.12 Geographic origin or cutaneous subtype (i.e. limited or diffuse cutaneous disease) did not affect the relationship between GI involvement and HRQoL. The global impact of GI involvement on SSc patients inde-pendent of ethnicity was affirmed in a recent study of 65 Chinese SSc patients and 127 age- and gender-matched controls.13 This study also demonstrated that the presence of distention, diarrhea, and constipation adversely affected social function and emotional well-being.13 Moreover, women with SSc suffer frequently not only from lower GI symptoms but also from pelvic floor symptoms.14 Pelvic pain, fecal and urine incontinence, as well as constipation and diarrhea were frequently present and significantly affected HRQoL in one study of 160 female SSc patients from the Mayo Clinic in Arizona.14 Interestingly, pelvic symptoms were also strongly associated with depression, which in turn was associated with diminished quality of life. The direct effect of pelvic symptoms on quality of life remained significant even after controlling for the pres-ence of depression among these patients. 14

\section{Effects of Gl involvement on mortality in SSc}

GI involvement not only severely affects quality of life, but it is also one of the leading causes of death in the SSc patients over the past five decades.7,15,16 An analysis of the Pittsburgh demonstrated that severe GI dysfunction affected 
$8 \%$ of patients with diffuse cutaneous SSc, and the presence of severe GI dysfunction was associated with an $85 \%$ mortality rate within 9 years of the diagnosis of severe GI involvement.7,17 Severe GI involvement was, in this study, defined as malabsorption syndrome, repeated episodes of intestinal pseudo-obstruction, or severe GI problems which required hyperalimentation; diarrhea responsive to antibi-otics without malnutrition, bloating after meals, and esoph-ageal stricture if associated with a $>10 \%$ weight loss or hospitalization. 17

These findings are consistent with the results of a more recent, large $(\mathrm{N}=556)$, multi-center, international inception cohort of SSc patients with a disease duration <2 years. 9 Severe GI disease (defined as present if a physician reported the presence of malabsorption, the need for hyperalimentation, one or more episodes of pseudo-obstruction, and/or a $>10 \%$ weight loss in association with the use of antibiotics for SIBO within the last year or esophageal stricture) was present in 5\% of all SSc patients at baseline and $8 \%$ went on to develop severe GI disease during the mean follow-up time of 4.3 years. The presence of severe GI involvement was associated with more than twofold increase in the risk of death, even after adjusting for age and gender.9 Age was also independently associated with the risk of death, whereas female sex was protective and associated with improved survival compared with male sex. Further supporting the strong association between severe GI involvement and mortality across SSc subtypes and geographic areas, a study of a large SSc cohort $(\mathrm{N}=349)$ from Singapore found that the use of parenteral nutrition was also a strong independent predictor of mortality.18

GI involvement remains one of the leading causes of deaths related to SSc over decades.7,16 Primary causes of death for SSc patients observed during a 30-year time were evaluated in 2007. From 1972 to 2002, the frequency of deaths due to GI involvement in SSc changed numerically, decreasing from $12 \%$ to $4 \%$ of all deaths, but this was not a statistically significant decrease. 7 The change in the rela-tive frequencies of causes of deaths due to GI involvement during this time is shown in Figure 2.7 In 2017, causes of death of SSc patients $(n=1072 / 11193,9.6 \%)$ between 2000 and 2011 enrolled in the international European Scleroderma Trials and Research (EUSTAR) database were examined.16 The frequency of deaths due to GI involvement was 3.5\% (Figure 2) and occurred more often in patients with diffuse cutaneous disease $(6 \%)$ compared with limited cutaneous disease (2\%), although this differ-ence was not statistically significant.16 The most common cause of GI death was complications due to intestinal involvement (6.6\%), followed by esophageal, gastric, and duodenal involvement $(1.1 \%)$.

It is important to highlight that, in the two aforementioned studies, the definition of death due to GI involvement differed. While in the Pittsburgh cohort death caused by GI involvement was defined as death due to malabsorption,

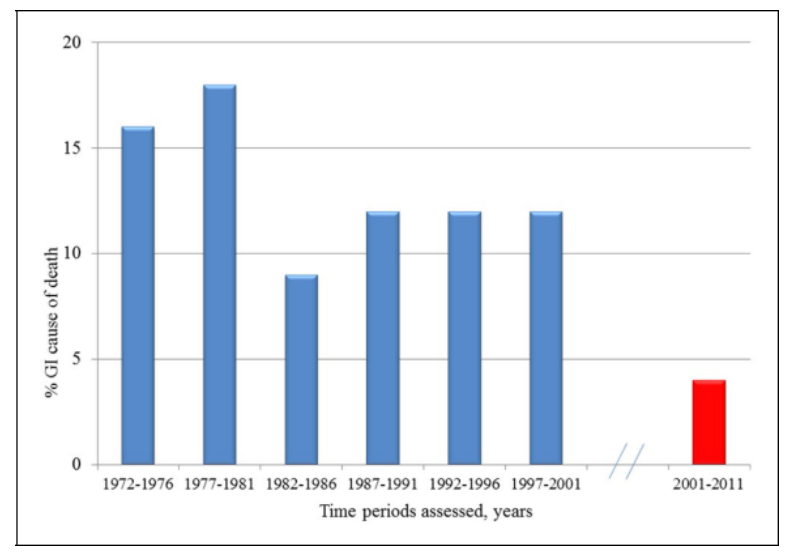

Figure 2. Causes of deaths in systemic sclerosis patients from the Pittsburg cohort (in blue) between 1972 and 2002 and the EUSTAR database (in red) from 2001 to 2011.

The results are presented as percentage of deaths caused by gastroin-testinal disease.

malnutrition, or hyperalimentation, in the EUSTAR database, death due to GI disease was defined as death from GI hemorrhage or obstruction.7,16 It is therefore possible that the estimates of mortality due to GI causes may be underestimated because SSc patients may die from other causes that are an indirect consequence of severe GI disease (i.e. aspiration pneumonia due to severe GERD, sepsis due to a central line infection in a patient receiving parenteral nutrition).

\section{Conventional treatment for GI symptoms in SSc}

Treatment for GI involvement in SSc is presently limited to symptom relief and does not adequately address the underlying problem. Improved therapies in SSc are therefore highly needed.19 Lifestyle modification is often necessary for SSc patients with GI involvement, and these modifications include the following: altering their diet, eating smaller meals more frequently, not eating for sev-eral hours before lying down, and avoiding high fiber, high fat foods, as well as abstaining from smoking tobacco and consuming alcohol. Elevating the head of the bed is also important for SSc patients, especially for patients with upper GI involvement.20 An expert consensus from the UK Scleroderma study group for the management of GI dis-ease made the following broad statements: (1) all patients should have anti-reflux treatment with a proton pump inhibitor (PPI) or histamine receptor 2 (H2) blocker and antacids; (2) midgut disease might lead to bacterial over-growth that responds to antibiotics; (3) prokinetics and dietary adjustment might alleviate abdominal distension;

(4) enteral or parenteral nutrition should be considered in cases of refractory weight loss; and (5) anorectal disease needs specialist investigations.1,21 Of note, the side-effects associated with the long-term use of these agents must be 
weighed against their purported efficacy. For subclinical acid reflux (i.e. acid reflux identified by manometry in an asymptomatic patient), PPI use should still be considered to prevent Barrett's esophagitis even though PPIs may increase the risk of small intestine bacterial overgrowth. An overview of commonly used medications for the treat-ment of GI manifestations in SSc and a comprehensive description including other approaches for treatment can be found elsewhere.1,3,10

\section{New approaches to therapy}

\section{Immune-modulating approaches}

Although vasculopathy and fibrosis are generally considered the key pathological drivers for GI disease in SSc, this dimension of SSc is generally not considered as immuneresponsive. However, immune-based therapies have not been adequately investigated as therapeutic options for SScGI involvement, and recent studies suggest that these therapies may be beneficial based on our evolving understanding of the pathobiology of SSc-GI involvement. These assumptions are mainly based on the following: first, there is growing evidence that the presence of certain antibodies is associated with GI involvement in SSc (i.e. anti-RNPC3 antibodies, as mentioned previously). Moreover, SSc patients with severe GI involvement had a higher prevalence of antibodies against muscarinic-3 acetylcholine receptor (anti-M3R antibody), suggesting that M3R-mediated enteric cholinergic neurotransmission may provide a pathogenic mechanism for GI dysmotility in SSc.22 Second, immunomodulatory strategies, for GI disease in SSc, are also supported by the finding that hematopoietic stem cell transplantation (HSCT) improves outcomes in multiple organ systems in SSc.23,24

It is important to distinguish between ongoing disease activity and organ damage leading to GI symptoms when determining whether immunosuppression is indicated. Based on the aforementioned studies, a potential disease-modifying strategy for active SSc with GI involvement includes the administration of intravenous immunoglobu-lin (IVIG) treatment. In a case series study $(n=15)$, IVIG treatment showed an improvement in various clinical aspects of SSc, including GI disease.25 All of the SSc patients in this study had active inflammatory myopathy that was unresponsive to conventional disease-modifying agents. The patients were treated with IVIG (mean 2.3 years of IVIG treatment) at a frequency ranging from every 6 weeks to every 4 months. The results demonstrated a reduction in gastroesophageal reflux frequency and severity and an improvement in the Gastrointestinal Tract (GIT) 2.0 scores over the course of the study. The authors concluded that the sustained benefit from IVIG treatment may suggest a specific potential benefit for immunomodu-lation in SSc patients with established GI complications.
However, prospective and randomized controlled trials (RCTs) designed to assess the effect of IVIG on GI manifestations are needed to further evaluate the effects of IVIG treatment on GI involvement in SSc.

\section{GI microbiota targets}

Accumulating evidence suggests that alterations in GI microbiota are a defining feature of patients with SSc.26,27 The first study to explore the GI microbiome in SSc $(n=17)$ demonstrated that patients with SSc had an altered microbiota composition compared with healthy age- and gender-matched controls in an analysis of colonic lavage specimens.28 We subsequently compared the microbiota of two independent SSc cohorts (UCLA and OUH, $n=34$ ) and found similar alterations in GI microbiota of SSc patients compared with controls in an analysis of stool specimens.29 Similar findings were also reported by in an Italian SSc cohort including $59 \mathrm{SSc}$ patients. 30 Cross-sectional data from a relatively large Swedish SSc cohort $(\mathrm{n}=98)$ provided further evidence of an association between GI microbiome alterations and GI symptoms.31 This study also found that fecal calprotectin levels were higher in SSc patients with GI dysbiosis compared with SSc patients without dysbiosis, suggesting that inflamma-tion may moderate the relationship between GI microbiota alterations and GI symptoms in SSc.31 These studies have demonstrated that specific bacterial genera and species are associated with improvement or worsening in GI symp-toms, suggesting that a possible strategy for alleviating GI symptoms in SSc could target selective augmentation or elimination of specific bacterial species.

To date, it is unknown whether the divergent microbiota is a consequence of the disease or represents a causative factor; but comparative studies in murine models have demonstrated that gut microbiota constituents may impair peristalsis leading to dysmotility and GI complications. 32 Fecal microbiota transplantation (FMT) is undergoing investigation as a potential therapeutic intervention for several other diseases showing a good safety profile and modest clinical effects; however, it had not been assessed in rheumatic diseases, including SSc.33,34 Based on these observations, we hypothesized that manipulation of the GI microbiome by a standardized FMT could have beneficial effect on GI symptoms and GI microbiota composition also in patients with SSc. A pilot study on FMT in $10 \mathrm{SSc}$ patients with commercially available anaerobic cultivated human intestinal microbiota (ACHIM) demonstrated that FMT was associated with lower GI symptoms and altered fecal microbial composition (Figure 3).35 It also seemed that acquisition of a different gut microbiota induced immunological changes, as reflected by alterations in the pattern and/or extent of IgA and IgM coating. 35 Importantly, although the direct FMT-related side-effects were mild and transient, two severe adverse events related to fecal 


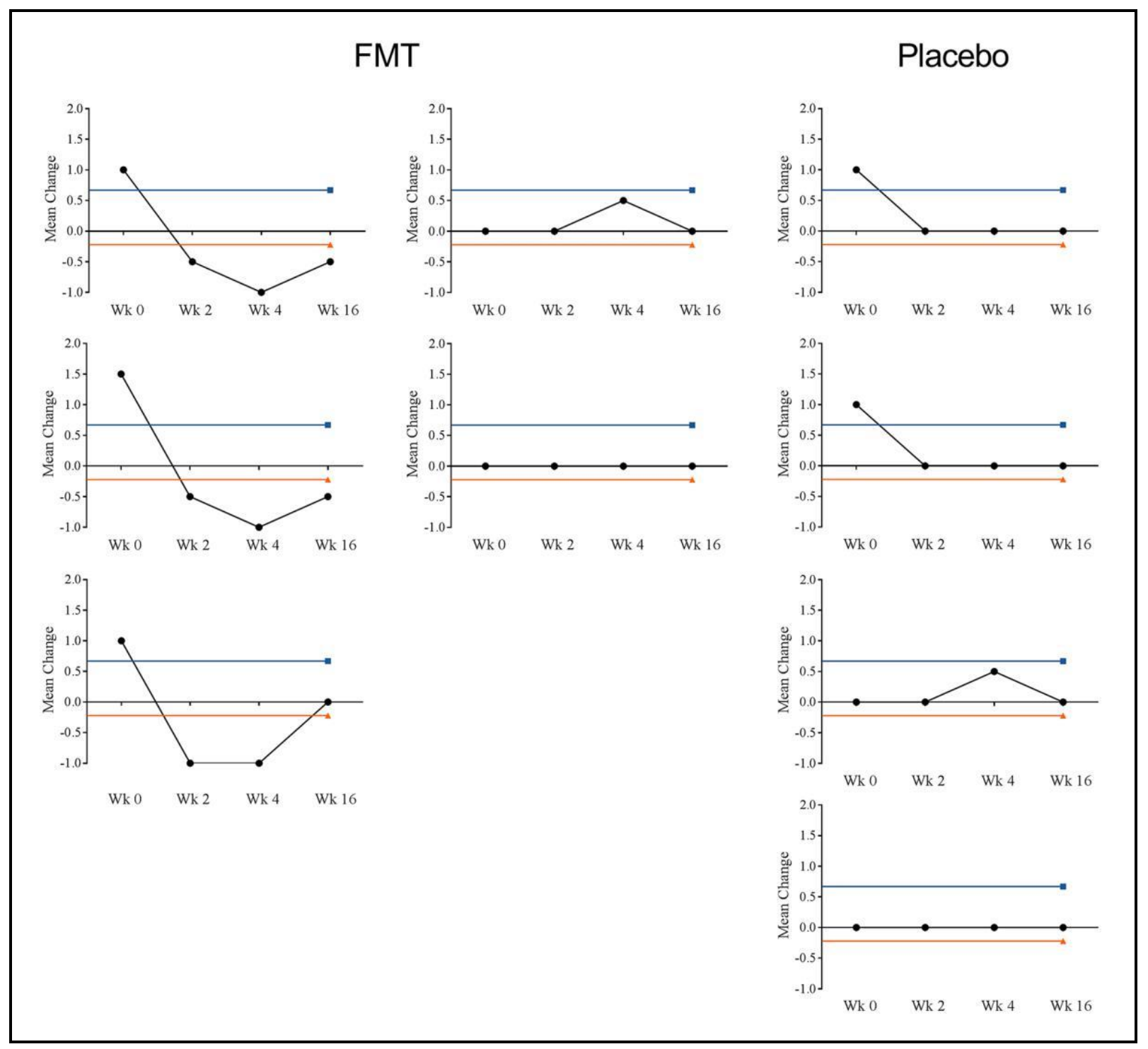

Figure 3. Evolution of the UCLA GIT score from week 0 to week 16 after two infusions of gut microbiota at week 0 and week 2. Three patients in the fecal microbiota transplantation (FMT) group (left panel) had apparent diarrhea at baseline, shown as values above the blue line marking clinical meaningful diarrhea, and two patients in the placebo group (right panel, FMT group). All patients treated with FMT showed a clinical meaningful effect in the diarrhea score, shown as values below the orange line marking the minimal clinical important difference measured by the UCLA GIT 2.0 score, while none of the placebo group showed an improvement.

microbiota transfer by gastroduodenoscopy were encountered. One control participant developed laryngospasms at the first gastroduodenoscopy necessitating study exclu-sion, while another control participant experienced duode-nal perforation during gastroduodenoscopy at the last study visit. Also, there has been recent concern regarding the safety of FMT after two fatal deaths in the United States, due to donor feces containing drug-resistant E. coli. However, the ACHIM used in the pilot trial does not con-tain resistant bacteria as tested on a regular basis.

Probiotics could potentially modulate the microbiome and the immune system in SSc. An RCT including 73 patients, receiving either probiotics $(n=37)$ or placebo $(n=36)$, evaluated the efficacy of probiotics on GI symptoms and immune responses in SSc patients.36 After 8 weeks, no difference in the UCLA GIT 2.0 score between the two groups was observed, but the probiotic group showed a significant decrease in the proportion of Th17 cells compared with placebo. No severe side-effects were observed in this study.36 Of note, there have been some concerns regarding the safety of probiotic use in immunocompromised patients developing life-threatening pneumonia, endocarditis, and sepsis due to some probi-otic strains becoming opportunistic pathogens.37 To our knowledge, there are no reports regarding safety issues associated with probiotic use in SSc patients to date. 


\section{Surgical approaches}

Surgical management of severe GERD may be an option for some patients with SSc who fail medical management.38 A recent study demonstrated that both gastric bypass and to a less extent fundoplication surgery led to an improvement or resolution in reflux symptoms in a small cohort of patients with SSc $(\mathrm{n}=14) .39$ Future studies are needed to assess long-term outcomes in patients undergo-ing these surgeries.

\section{Future perspectives}

The studies above demonstrate that morbidity and mortal-ity outcomes related to SSc-GI involvement have not significantly improved over time. This is likely a direct result of our limited understanding of the pathogenesis of GI dysfunction in SSc. Emerging evidence suggests that autoantibody production and alterations in GI microbiota may contribute to GI involvement in SSc, but further research is needed to understand the mechanisms by which these alterations affect disease progression. Also, all future clinical trials should include measures of GI disease activity and damage, especially since GI involvement has such a profound effect on HRQoL. The development of more objective SSc-specific GI measurement tools that are sensitive to change is also greatly needed.

Treatment for SSc-related GI manifestations represents a major unmet area of research. Despite efforts to alleviate GI symptoms in SSc, no curative or disease-modifying therapies exist for managing this dimension of SSc. Moreover, even though the GI tract is the second most common organ system involved in SSc, and patients are affected by GI symptoms on a daily basis, there is a general reluctance to engage in a dialogue about these symptoms in clinical practice. Improved awareness of the GI burden in SSc and closer collaboration between specialists (e.g. gastroenterology, rheumatology, nutritionist, physiotherapy), combined with a more problem-orientated approach, will hopefully lead to improved GI-related health outcomes in patients with SSc in the future.

In summary, the GI tract still remains a largely unexplored area of preclinical and clinical research in SSc. In order to improve morbidity and mortality outcomes in patients with SSc-GI involvement, more work is needed to (1) understand the cause(s) of SSc-GI involvement; (2) determine how to properly evaluate the extent and progression SSc-GI involvement; and (3) identify novel treatment targets for therapeutic intervention. Our ongoing research efforts evaluating the GI microbiome in SSc may help to illuminate the path forward toward understanding how to treat this troubling dimension of SSc.

\section{Funding}

The author(s) disclosed receipt of the following financial support for the research, authorship, and/or publication of this article: AMHV: has received research funding and/or consulting fees or other remuneration from Actelion, Boehringer Ingelheim and Roche. EV: has received research funding and/or consulting fees or other remuneration from Boehringer Ingelheim.

\section{References}

1. Denton CP and Khanna D. Systemic sclerosis. Lancet 2017; 390(10103): 1685-1699.

2. Thoua NM, Bunce C, Brough G, et al. Assessment of gastrointestinal symptoms in patients with systemic sclerosis in a UK tertiary referral centre. Rheumatology 2010; 49(9): 1770-1775.

3. Shreiner AB, Murray C, Denton C, et al. Gastrointestinal manifestations of systemic sclerosis. J Scleroderma Relat Disord 2016; 1(3): 247-256.

4. Schmeiser T, Saar P, Jin D, et al. Profile of gastrointestinal involvement in patients with systemic sclerosis. Rheumatol Int 2012; 32(8): 2471-2478.

5. Emmanuel A. Current management of the gastrointestinal complications of systemic sclerosis. Nat Rev Gastroenterol Hepatol 2016; 13(8): 461-472.

6. Ebert EC. Esophageal disease in scleroderma. J Clin Gastroenterol 2006; 40(9): 769-775.

7. Steen VD and Medsger TA. Changes in causes of death in systemic sclerosis, 1972-2002. Ann Rheum Dis 2007; 66(7): 940-944.

8. Lepri G, Guiducci S, Bellando-Randone S, et al. Evidence for oesophageal and anorectal involvement in very early sys-temic sclerosis (VEDOSS): report from a single VEDOSS/ EUSTAR centre. Ann Rheum Dis 2015; 74(1): 124-128.

9. Richard N, Hudson M, Wang M, et al. Severe gastrointestinal disease in very early systemic sclerosis is associated with early mortality. Rheumatology 2019; 58(4): 636-644.

10. McMahan ZH, Paik JJ, Wigley FM, et al. Determining the risk factors and clinical features associated with severe gastrointestinal dysmotility in systemic sclerosis. Arthritis Care Res 2018; 70(9): 1385-1392.

11. McMahan ZH, Domsic RT, Zhu L, et al. Anti-RNPC3 (U11/ U12) antibodies in systemic sclerosis are associated with moderate to severe gastrointestinal dysmotility. Arthritis Care Res 2019; 71(9): 1164-1170.

12. Frantz C, Avouac J, Distler O, et al. Impaired quality of life in systemic sclerosis and patient perception of the disease: a large international survey. Semin Arthritis Rheum 2016; 46(1): 115-123.

13. Yang H, Xu D, Li MT, et al. Gastrointestinal manifestations on impaired quality of life in systemic sclerosis. J Dig Dis 2019; 20: 256-261.

14. Umar SB, Griffing L, Garcia H, et al. The impact of pel-vic floor and lower gastrointestinal symptoms on quality of 
life in women with systemic sclerosis. J Clin Gastroenterol 2016; 50(6): e55-e59.

15. Wigley FM and Boin F. Clinical features and treatment of scleroderma A2. In: Firestein GS, Budd RC, Gabriel SE, et al. (eds) Kelley and Firestein's textbook of rheumatology. 10th ed. Philadelphia, PA: Elsevier, 2017, pp. 1424.e5-1460.e5.

16. Elhai M, Meune C, Boubaya M, et al. Mapping and predicting mortality from systemic sclerosis. Ann Rheum Dis 2017; 76(11): 1897-1905.

17. Steen VMTJ. Severe organ involvement in systemic sclerosis with diffuse scleroderma. Arthritis Rheum 2000; 43(11): 2437-2444.

18. Santosa A, Tan CS, Teng GG, et al. Lung and gastrointestinal complications are leading causes of death in SCORE, a multi-ethnic Singapore systemic sclerosis cohort. Scand J Rheumatol 2016; 45(6): 499-506.

19. Frech TM and Mar D. Gastrointestinal and hepatic disease in systemic sclerosis. Rheum Dis Clin North Am 2018; 44(1): 15-28.

20. Nagaraja V, McMahan ZH, Getzug T, et al. Management of gastrointestinal involvement in scleroderma. Curr Treatm Opt Rheumatol 2015; 1(1): 82-105.

21. Hansi N, Thoua N, Carulli M, et al. Consensus best prac-tice pathway of the UK scleroderma study group: gastrointestinal manifestations of systemic sclerosis. Clin Exp Rheumatol 2014; 32(6 Suppl. 86): S214-S221.

22. Kawaguchi Y, Nakamura Y, Matsumoto I, et al. Muscarinic3 acetylcholine receptor autoantibody in patients with systemic sclerosis: contribution to severe gastrointestinal tract dysmotility. Ann Rheum Dis 2009; 68(5): 710-714.

23. Sullivan K, Goldmuntz E, Keyes-Elstein L, et al. Myeloablative autologous stem-cell transplantation for severe scleroderma. N Engl J Med 2018; 378(1): 35-47.

24. van Laar JM, Farge D, Sont JK, et al. Autologous hematopoietic stem cell transplantation vs intravenous pulse cyclophosphamide in diffuse cutaneous systemic sclerosis. JAMA 2014; 311(24): 2490-2498.

25. Raja J, Nihtyanova SI, Murray CD, et al. Sustained benefit from intravenous immunoglobulin therapy for gastrointesti-nal involvement in systemic sclerosis. Rheumatology 2016; 55(1): 115-119.

26. Volkmann ER. Intestinal microbiome in scleroderma: recent progress. Curr Opin Rheumatol 2017; 29(6): 553-560.
27. Bellocchi $\mathrm{C}$ and Volkmann ER. Update on the gastrointestinal microbiome in systemic sclerosis. Curr Rheumatol Rep 2018; 20(8): 49.

28. Volkmann ER, Chang YL, Barroso N, et al. Systemic sclerosis is associated with a unique colonic microbial consortium. Arthritis Rheumatol 2016; 68(6): 1483-1492.

29. Volkmann ER, Hoffmann-Vold A-M, Chang Y-L, et al. Systemic sclerosis is associated with specific alterations in gastrointestinal microbiota in two independent cohorts. BMJ Open Gastroenterol 2017; 4(1): e000134.

30. Bellocchi C, Fernandez-Ochoa A, Montanelli G, et al. Microbial and metabolic multi-omic correlations in systemic sclerosis patients. Ann N Y Acad Sci 2018; 1421(1): 97-109.

31. Andreasson K, Alrawi Z, Persson A, et al. Intestinal dysbiosis is common in systemic sclerosis and associated with gas-trointestinal and extraintestinal features of disease. Arthritis Res Ther 2016; 18(1): 278.

32. Husebye E, Hellstrom PM, Sundler F, et al. Influence of microbial species on small intestinal myoelectric activity and transit in germ-free rats 2001; 280(3): G368-G380.

33. Paramsothy S, Kamm MA, Kaakoush NO, et al. Multidonor intensive faecal microbiota transplantation for active ulcerative colitis: a randomised placebo-controlled trial. Lancet 2017; 389(10075): 1218-1228.

34. Leffler DA and Lamont JT. Clostridium difficile infection. N Engl J Med 2015; 372(16): 1539-1548.

35. Hoffmann-Vold AFH, Chung BK, Didriksen H, et al. Fecal microbiota transplantation in systemic sclerosis: a doubleblind, placebo-controlled randomized pilot trial. Ann Rheum Dis 2019(78): 246-247.

36. Marighela TF, Arismendi MI, Marvulle V, et al. Effect of probiotics on gastrointestinal symptoms and immune parameters in systemic sclerosis: a randomized placebocontrolled trial. Rheumatology 2019; 58: 1985-1990.

37. Kothari D, Patel S and Kim SK. Probiotic supplements might not be universally-effective and safe: a review. Biomed Pharmacother 2019; 111: 537-547.

38. Bakhos CT, Petrov RV, Parkman HP, et al. Role and safety of fundoplication in esophageal disease and dysmotility syndromes. J Thorac Dis 2019; 11(Suppl. 12): S1610-S1617.

39. Yan J, Strong A, Sharma G, et al. Surgical management of gastroesophageal reflux disease in patients with systemic sclerosis. Surg Endosc 2018; 32(9): 3855-3860. 